\title{
Efecto de una Intervención Educativa en Enfermería sobre el Nivel de Conocimiento del programa NIDCAP.
}

\author{
Effect of a Nursing Educational Intervention on the Level of Knowledge of the NIDCAP \\ program
}

\author{
Luis U. Ortiz-Mendoza ${ }^{a}$, Rosa M. Baltazar-Téllez, ${ }^{b}$ Alma B. Tapia-García ${ }^{c}$, Alma B. López- \\ Escudero ${ }^{d}$ y José Arias-Rico ${ }^{e}$
}

\begin{abstract}
:
There is a trend in the care of premature newborns called "Care focused on development and the family", within which is the NIDCAP program involves medical and nursing interventions focused on reducing the stress and suffering of the child, to promote neurological and emotional development and facilitate family integration. The present study aims to determine the effect of an educational intervention on the level of knowledge of the NIDCAP program in nursing personnel, using a quasi-experimental, quantitative, descriptive, longitudinal design. This study was made in Hospital Regional del Valle del Mezquital, where an evaluation instrument was applied regarding the NIDCAP program to 12 nurses from the NICU in different shifts. Prior to the implementation of the educational intervention, the nursing staff had a low level of knowledge; the post-test, the result was an improvement of knowledge into a higher level. Three months later, in the evaluation stage, the level of knowledge remained high. In conclusion, there was a significant effect of the educational intervention from the pre-test, post-test and the level of knowledge of the participants about the NIDCAP program.
\end{abstract}

Keywords:

Care, NIDCAP; Premature; Intervention; Educational

Resumen:

Existe una corriente en el cuidado de los recién nacidos prematuros denominada, "Cuidados centrados en el desarrollo y la familia", dentro de la cual se encuentra el programa NIDCAP que involucra una serie de intervenciones médicas y de enfermería para disminuir el estrés y sufrimiento del niño, favorecer el desarrollo neurológico, emocional y facilitar la integración de la familia. El presente estudio tiene como objetivo determinar el efecto de una intervención educativa sobre el nivel de conocimientos del programa NIDCAP en el personal de enfermería, utilizando el diseño cuasiexperimental, de tipo cuantitativo, descriptivo, longitudinal. Se realizó en el Hospital Regional del Valle del Mezquital en donde se aplicó un instrumento de evaluación respecto al programa NIDCAP a 12 enfermeros de la UCIN en distintos turnos. Previo a la implementación de la intervención educativa el personal de enfermería tenía un nivel de conocimiento bajo; posteriormente en el postest se obtuvo como resultado que el total de la muestra mejoró a un nivel de conocimiento alto. Tres meses después en etapa de evaluación se observó que continúo alto el nivel de conocimientos. En conclusión, se observó que existe un efecto significativo de la intervención educativa desde el pre test, post test y evaluación con respecto al nivel de conocimiento de los participantes sobre el programa NIDCAP.

Palabras Clave:

Cuidado; NIDCAP; Prematuro; Intervención; Educativa

\footnotetext{
a Autor de Correspondencia, Universidad Autónoma del Estado de Hidalgo, ORCID: 0000-0002-3837-6885, Email: mendozaom27@gmail.com

b Universidad Autónoma del Estado de Hidalgo, ORCID: 0000-0002-2168-7564, Email: rosa_baltazar@uaeh.edu.mx

c Universidad Autónoma del Estado de Hidalgo, ORCID: 0000-0001-5312-5147 Email: ta368795@uaeh.edu.mx

d Universidad Autónoma del Estado de Hidalgo, ORCID: 0000-0003-4578-6441, Email: 10315896@uaeh.edu.mx

e Universidad Autónoma del Estado de Hidalgo, ORCID: 0000-0003-0219-0410, Email: josearias.rico@ hotmail.com
} 


\section{Introducción}

La Organización Mundial de la Salud (OMS) define que son prematuros aquellos recién nacidos (RN) que nacen antes de completar las 37 semanas de gestación, considera que la prematuridad es la principal causa de mortalidad entre los $\mathrm{RN}{ }^{1}$

De acuerdo con la literatura mundial, la incidencia de prematurez se estima entre $5 \%$ y $10 \%$, aunque en países subdesarrollados es más alta. En México del 2015 al 2017, el Instituto Nacional de Perinatología reporta una incidencia de $19.7 \%$ de recién nacidos prematuros, mientras que el Instituto Mexicano del Seguro Social (IMSS) describe incidencias que varían desde el $2.8 \%$ hasta el $16.6 \%$ de prematurez. ${ }^{2}$

En las últimas décadas, la supervivencia de los niños prematuros ha mejorado, fundamentalmente, gracias al traslado neonatal, corticoides prenatales, el surfactante y a mejores recursos tecnológicos. Sin embargo, esto no se ha acompañado de una disminución tan significativa de las secuelas a largo plazo, ya que el nacimiento antes de término interrumpe el desarrollo del sistema nervioso central en un momento de crecimiento rápido y vulnerable del mismo. ${ }^{3}$

Actualmente, existe una corriente en el cuidado de los recién nacidos prematuros, denominada Cuidados Centrados en el Desarrollo y la Familia (CCD). Se basan en la interacción dinámica entre recién nacido, familia y ambiente, que aplica al periodo neonatal, intentando optimizar el macroambiente (ruidos, luces) y el microambiente (postura, manipulaciones, dolor) en el que se desarrolla el niño, $y$, por supuesto, implica a la familia para potenciar su papel de cuidador principal, de una manera activa y continuada. ${ }^{3,4}$

Dentro de esta CCD, se encuentra uno de los modelos más extendidos de aplicación, denominado Programa de Evaluación y Cuidado Individualizado del Desarrollo del Recién Nacido (NIDCAP), que consiste en una serie de intervenciones médicas y de enfermería dirigidas a disminuir el estrés, el sufrimiento del niño, a favorecer su desarrollo neurológico, emocional y a facilitar la integración de los miembros de la familia como cuidadores del niño. ${ }^{5}$

La implementación de estos cuidados comporta un cambio importante en las rutinas de trabajo. Los procesos se adaptan a las características del niño, de su familia, y no al contrario. El programa NIDCAP supone un cambio en las actitudes que los profesionales deben adoptar en relación a los pacientes. 6

El personal de enfermería que trabaja en el servicio de Unidad de Cuidados Intensivos Neonatales (UCIN), día a día se ve reflejada una debilidad en los conocimientos sobre el cuidado del neurodesarrollo del recién nacido prematuro. Esto nos hace reflexionar, que gran parte del personal no ha unificado criterios sobre los cuidados que se deben tener a nivel neuronal del recién nacido prematuro.7

\section{Método}

\section{Diseño y tipo de investigación}

Se utilizó un diseño cuasiexperimental, de tipo descriptivo/longitudinal, con una técnica de aplicación de pre test y pos test.

\section{Población y muestra}

Se realizó en el Hospital Regional del Valle del Mezquital, con una población de 128 enfermeras (os).

Tipo de muestreo: No Probabilístico.

Criterios de inclusión: la $n=$ estuvo constituida por 12 enfermeras (os) adscritos al servicio de Unidad de Cuidados Intensivos Neonatales de los diferentes turnos de jornada laboral.

\section{Instrumento}

El programa de intervención educativa estuvo conformado por 8 sesiones con duración de 30 minutos cada una.

Para la recolección de datos de utilizó el instrumento "Nivel de Conocimiento de las Enfermeras sobre el Cuidado Centrado en el Desarrollo del Prematuro", el cual, consta de 2 partes, la primera contiene datos personales y referenciales, la segunda parte incluye aspectos a evaluar con 20 preguntas divididas en tres dimensiones, presentan 4 alternativas de respuesta, asignándose 1 punto por cada respuesta correcta y 0 por la respuesta incorrecta, obteniendo así un puntaje máximo de 20 puntos. La categorización de los resultados está basada en la escala siguiente:

Nivel de conocimiento alto: 16 a 20 puntos.

Nivel de conocimiento medio: 11 a 15 puntos.

Nivel de conocimiento bajo: 0 a 10 puntos.

\section{Procedimiento}

La recolección de datos se llevó a cabo mediante 3 etapas; en la primera etapa (Pre test) se aplicó el instrumento para determinar el nivel de conocimientos de cada participante.

Segunda etapa, intervención educativa, se impartieron sesiones educativas en distintos turnos laborales, denominadas "Intervenciones con el Programa de Evaluación y Cuidado Individualizado del Desarrollo del Recién Nacido NIDCAP", abarcando un periodo de tres meses. Para finalizar.

Tercera etapa, se realizó el post test, a través del mismo instrumento, con el objetivo de evaluar el impacto del 
programa de capacitación y determinar el nivel de conocimiento del Programa de Evaluación y Cuidado Individualizado del Desarrollo del Recién Nacido NIDCAP.

Tres meses después se volvió a aplicar el instrumento, para determinar el nivel de conocimiento que poseían los participantes y evaluar si fue significativo el conocimiento adquirido.

\section{Análisis estadístico}

Se utilizó el paquete estadístico para las ciencias sociales IBM, SPSS versión 23 en español. Se utilizó estadística descriptiva, la cual fue representada en tablas de distribución de frecuencias y porcentajes de cada una de las variables.

\section{Resultados}

De acuerdo con los resultados se encontró que, de la muestra total (12) el promedio de edad fue de 35.2 años ( $D E=8.8$ ), el valor mínimo de edad fue de 26 años y el máximo de 50 años. En antigüedad en el servicio la media fue de 8.2 años, (DE=8.2) el valor mínimo fue de 1 año de antigüedad, y el máximo de 26 de años.

En la Figura 1 se observa que más de la mitad de la muestra $(66.7 \%)$ mencionaron conocer el programa NIDCAP, mientras que una tercera parte (33.3\%) mencionaron no saber a qué se refiere este programa.

Figura 1. Conoce el programa NIDCAP.

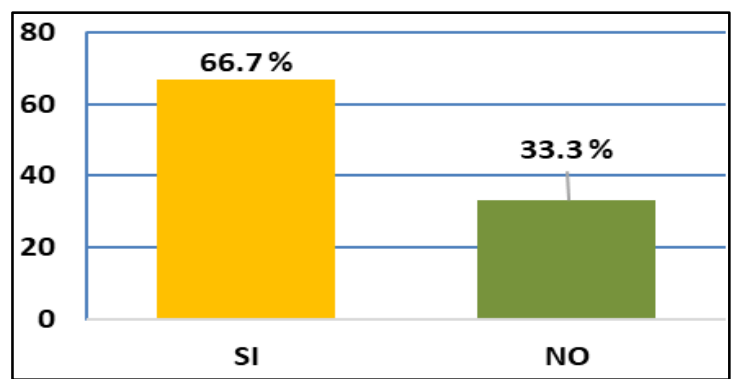

Fuente: Cuestionario "Nivel de conocimiento de la enfermera sobre el cuidado centrado en el desarrollo del prematuro". $n=12$

En la Figura 2 se observa que en la aplicación del pre test, tres cuartas partes (75\%) del personal obtuvieron un nivel de conocimiento bajo respecto al programa NIDCAP, posterior a la intervención educativa se aplicó el post test, donde el $100 \%$ del personal obtuvo un nivel de conocimiento alto respecto al programa NIDCAP, tres meses después de la aplicación del post test se aplicó la evaluación donde el $100 \%$ obtuvo un nivel de conocimiento alto respecto al programa NIDCAP.
Figura 2. Nivel de conocimiento de los participantes sobre el programa NIDCAP

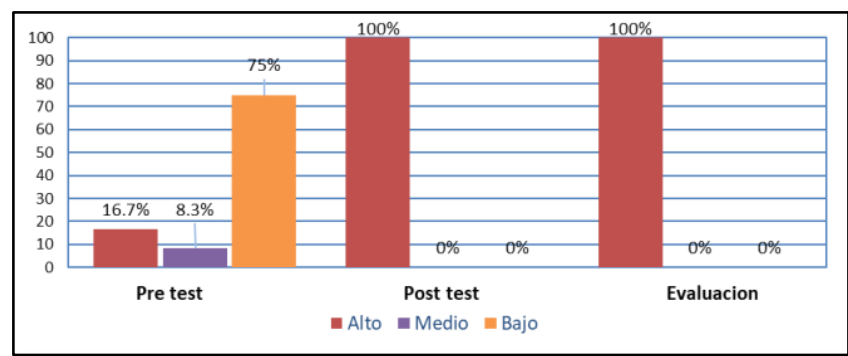

Fuente: Cuestionario "Nivel de conocimiento de la enfermera sobre el cuidado centrado en el desarrollo del prematuro".

\section{Discusión}

El principal objetivo de la investigación fue evaluar el efecto de una intervención educativa en el personal de enfermería, respecto al nivel de su conocimiento sobre el programa NIDCAP. La intervención desarrollada tuvo un grupo experimental de 12 personas del cual se compararon los resultados obtenidos en el pre test, post test y evaluación.

Referente al conocimiento en el personal de enfermería sobre NIDCAP, el $66.7 \%$ respondieron conocer las intervenciones que comprende el programa NIDCAP, por el contrario, el $33.3 \%$ manifestaron no conocer dicho programa.

Mosqueda, et al. ${ }^{8}$ realizó una investigación con el objetivo de evaluar el nivel de impacto de un curso de capacitación para el cuidado del desarrollo sobre el conocimiento y la satisfacción de los profesionales de la salud en unidades neonatales, y dentro de sus hallazgos, la mayoría de los profesionales de la salud que trabajan en unidades neonatales en Madrid tienen un nivel aceptable de conocimiento de la atención del desarrollo del prematuro, similar a los resultados encontrados en este estudio.

Por otro lado, Mosqueda et al. ${ }^{8}$ y Borras, Ángel, Ros, Montejano, y Solaz ${ }^{9}$ refieren es sus resultados que posterior a una intervención educativa el nivel de conocimiento sobre programa NIDCAP mejoró en los participantes de manera significativa coincidiendo con el resultado de esta investigación.

\section{Conclusiones}

Con lo anterior, se concluye qué, un mayor porcentaje del personal de enfermería posee un nivel de conocimiento bajo respecto al programa NIDCAP previo a una intervención educativa, y que, posterior a ésta, se evidenció un efecto significativo, al incrementar el nivel de conocimiento del personal de salud.

Las intervenciones educativas son efectivas no sólo por el cuerpo de conocimiento que brinda el capacitador, sino 
por el conjunto de aprendizaje que aporta cada uno de los integrantes (aprendizaje social) en este caso el personal de enfermería, si las actividades son correctas y generan un cambio positivo de las personas, estas serán compartidas de profesional a profesional, se logrará la aceptación y posterior a ello, mejorará la calidad del cuidado del recién nacido, en las Unidades de Cuidados Intensivos Neonatales. ${ }^{10}$

\section{Referencias}

[1] Organización Mundial de la Salud. Nacimientos prematuros [Internet]. Ginebra; OMS; 2018 [citado 2021 Mar 16]. Disponible en: https://www.who.int/es/news-room/fact-sheets/detail/preterm-birth

[2] López-García Berenice, Ávalos Antonio Nilvia, Díaz Gómez Noé Belmar. Incidencia de prematuros en el Hospital General Naval de Alta Especialidad 2015-2017. Rev. sanid. mil. [Internet]. 2018 Feb [citado 2021 Mar 16]; 72(1): 19-23. Disponible en: http://www.scielo.org.mx/scielo.php?script=sci_arttext\&pid=S0301696X2018000100019\&lng=es. Epub 20-Ago-2019.

[3] Harillo Acevedo, F.D., Rico Bcerra, J.I. y López Martínez, Ángel 2017. La filosofía de los cuidados centrados en el desarrollo del recién nacido prematuro (NIDCAP): una revisión de la literatura. Enfermería Global [Internet]. 2017 Oct [citado 2021 Mar 16] 16(4): 577-602. Disponible en: DOI: https://doi.org/10.6018/eglobal.16.4.263721

[4] Contreras S. Cuidados centrados en el desarrollo bajo la perspectiva de enfermería en la unidad de cuidados intensivo neonatales de un Hospital de España y México.[Master's thesis]. España: Universidad de Oviedo; 2012. 51 p. Disponible en: http://digibuo.uniovi.es/dspace/bitstream/10651/4081/6/TFM_Guada Contreras.pdf

[5] Moro M. De Guardia en Neonatología. 3ra. ed. Editorial Panamericana; 2016.

[6] Arrollo G. Actualización en enfermería, una responsabilidad profesional. Rev Enferm IMSS [Internet]. 2002 [citado 2021 Mar 16] 10(1): 3-6. Disponible en: https://www.medigraphic.com/pdfs/enfermeriaimss/eim2002/eim021b.pdf

[7] Fernández Y., Funes M. y Ladino V. Importancia del rol de enfermería en el neurodesarrollo del recién nacido pretermino. [Tesina] Argentina: Universidad Nacional de Cuyo. 2015; 82 p. Disponible en: https://core.ac.uk/download/pdf/83116557.pdf

[8] Mosqueda R., Lora D., Pavón A., Ureta N., Moral M. y Pallás C. Impacto de un curso de capacitación para el cuidado del desarrollo sobre el conocimiento y la satisfacción de los profesionales de la salud en unidades neonatales. Pediatr Neonatol. [Internet]. 2016 Abr. [citado 2021 Mar 16] 57(2): 97-104. Disponible en: https://www.ncbi.nlm.nih.gov/pubmed/26205438

[9] Borras M., Ángel M., Ros R., Montejano R., y Solaz A. Efecto de la formación interna sobre cuidados centrados en el desarrollo en profesionales de Neonatología, Hospital Universitario Politécnico la $\mathrm{Fe}$, Valencia. Rev Enfer Doc. [Internet]. 2018 Ene-Jun [citado 2021 Mar 16] Ene-Jun(110); 4-10. Disponible en https://www.researchgate.net/profile/Rosario_Navarret

[10] Bautista R. (2020). Impacto de un programa educativo como estrategia para el control de la hipertensión arterial. [Master's Thesis]. México: Universidad Nacional Autónoma de México; 2019. 85 p. Disponible en: http://132.248.9.195/ptd2019/mayo/0789253/Index.html 